November 12, $2018 \quad 5: 28 \quad$ WSPC/INSTRUCTION $\quad$ FILE

International Journal of Quantum Information

(C) World Scientific Publishing Company

\title{
LIMITATIONS OF SOME SIMPLE ADIABATIC QUANTUM ALGORITHMS
}

\author{
LAWRENCE M. IOANNOU \\ Department of Applied Mathematics and Theoretical Physics, University of Cambridge, \\ Wilberforce Road \\ Cambridge, Cambridgeshire, CB3 OWA, United Kingdom \\ lmi22@cam.ac.uk \\ MICHELE MOSCA \\ Institute for Quantum Computing, University of Waterloo, 200 University Avenue West \\ Waterloo, Ontario, N2L 3G1, Canada \\ mmosca@iqc.ca \\ Perimeter Institute for Theoretical Physics, 31 Caroline Street North \\ Waterloo, Ontario, N2L 2Y5, Canada \\ Received Day Month Year \\ Revised Day Month Year
}

\begin{abstract}
Let $H(t)=(1-t / T) H_{0}+(t / T) H_{1}, t \in[0, T]$, be the Hamiltonian governing an adiabatic quantum algorithm, where $H_{0}$ is diagonal in the Hadamard basis and $H_{1}$ is diagonal in the computational basis. We prove that $H_{0}$ and $H_{1}$ must each have at least two large mutually-orthogonal eigenspaces if the algorithm's running time is to be subexponential in the number of qubits. We also reproduce the optimality proof of Farhi and Gutmann's search algorithm in the context of this adiabatic scheme; because we only consider initial Hamiltonians that are diagonal in the Hadamard basis, our result is slightly stronger than the original.
\end{abstract}

Keywords: adiabatic; quantum; bounds.

\section{Introduction}

Farhi et al $!^{1}$ proposed a continuous-time quantum algorithm for solving NPcomplete combinatorial search problems, invoking the adiabatic approximation. ${ }^{2}$ It has been shown that Farhi et al.'s original proposal for the 3SAT problem has exponential worst-case complexity $\stackrel{3}{3}$ However, the average-case performance of (possible modifications of) the original proposal is still an open question. $451617 \mathrm{~A}$ more general notion of adiabatic quantum computation (not considered here) is universal $\stackrel{8}{[}$

This work supplements the similar recent work ${ }^{9|10| 11}$ on analytic lower bounds on the runtime of certain classes of simple adiabatic quantum algorithms. Our as-

${ }^{a}$ previously appearing in Ref. 16 
sumptions are weaker: for our general bound, we do not assume that the initial Hamiltonian is a one-dimensional projector. Thus, the dimension of the secondlargest eigenspace of the Hamiltonians emerges as a factor in the algorithms' complexity.

Let the function $f:\{0,1\}^{n} \longrightarrow\{0,1\}$ such that

$$
f(z)= \begin{cases}1 & \text { if } z \neq w \\ 0 & \text { if } z=w\end{cases}
$$

encode a general search problem (with unique solution $w$ ) for which we seek a quantum algorithm. The uniform-amplitude superposition state,

$$
|u\rangle \equiv \frac{1}{\sqrt{N}} \sum_{z \in\{0,1\}^{n}}|z\rangle,
$$

will be the starting state (at $t=0$ ) for all the algorithms we consider. Define the Walsh-Hadamard transform (or just Hadamard transform)

$$
W=\frac{1}{\sqrt{N}} \sum_{x \in\{0,1\}^{n}} \sum_{y \in\{0,1\}^{n}}(-1)^{x \bullet y}|x\rangle\langle y| .
$$

Let

$$
|\bar{z}\rangle \equiv W|z\rangle, \quad \text { for all } z \in\{0,1\}^{n} .
$$

We define the Hadamard basis to be $\left\{|\bar{z}\rangle: z \in\{0,1\}^{n}\right\}$.

Recall from Ref. 12 and Ref. 13 that the continuous-time version of the general search problem, defined in Ref. 14, may be solved adiabatically with initial and final Hamiltonians

$$
H_{u}=\sum_{z \in\{0,1\}^{n}} h_{\operatorname{search}}(z)|\bar{z}\rangle\langle\bar{z}|
$$

and

$$
H_{w}=\sum_{z \in\{0,1\}^{n}} f(z)|z\rangle\langle z|,
$$

respectively, where $h_{\text {search }}(z) \in\{0,1\}$ and $h_{\text {search }}(z)=0 \Leftrightarrow z=0^{n}$.

If $f$ is the truth function corresponding to a Boolean formula (also denoted $f)$ which is an instance of the 3SAT problem 15 , then we can define the function $v:\{0,1\}^{n} \longrightarrow\{0,1, \ldots\}$,

$$
v(z) \equiv \text { the number of clauses of } f \text { violated by assignment } z \text {. }
$$

Recall that Farhi et al.'s original adiabatic algorithm for 3SAT uses initial and final Hamiltonians

$$
H_{\mathrm{I}}=\sum_{z \in\{0,1\}^{n}} h(z)|\bar{z}\rangle\langle\bar{z}|
$$


November $12, \quad 2018 \quad 5: 28 \quad$ WSPC/INSTRUCTION $\quad$ FILE

and

$$
H_{F}=\sum_{z \in\{0,1\}^{n}} v(z)|z\rangle\langle z|,
$$

respectively, where $h(z)=\sum_{i: z_{i}=1} d_{i}$ and $d_{i}$ is the number of clauses of $f$ that contain variable $z_{i}$.

\section{Results}

It is convenient to cast Farhi et al.'s adiabatic 3SAT algorithm in a more general notation. Let $N=2^{n}$. Let $p(n)$ and $q(n)$ be two nonnegative integer functions of $n$. Let $\mathcal{P}=\left\{P_{j}\right\}_{j=0,1, \ldots, p(n)}$ and $\mathcal{Q}=\left\{Q_{k}\right\}_{k=0,1, \ldots, q(n)}$ be two partitions of $\{0,1\}^{n}$, that is, the following two unions are disjoint:

$$
\bigcup_{k=0}^{q(n)} Q_{k}=\{0,1\}^{n}=\bigcup_{j=0}^{p(n)} P_{j} .
$$

Define the projectors

$$
\hat{Q}_{k} \equiv \sum_{z \in Q_{k}}|\bar{z}\rangle\langle\bar{z}| \quad \text { and } \quad \hat{P}_{j} \equiv \sum_{z \in P_{j}}|z\rangle\langle z|
$$

onto the eigenspaces of the two Hamiltonians $H_{0}$ and $H_{1}$,

$$
H_{0} \equiv \sum_{k=0}^{q(n)} F_{k} \hat{Q}_{k} \quad \text { and } \quad H_{1} \equiv \sum_{j=0}^{p(n)} E_{j} \hat{P}_{j},
$$

where the two sequences of energy eigenvalues $\left(E_{j}\right)_{j=0,1, \ldots, p(n)}$ and $\left(F_{k}\right)_{k=0,1, \ldots, q(n)}$ are strictly increasing sequences of real numbers. Assume further that $F_{0}=0=E_{0}$ and that the starting state $|u\rangle$ is a ground state of $H_{0}$, i.e. $0^{n} \in Q_{0}$. We will also write

$$
H_{0}=\sum_{z \in\{0,1\}^{n}} F(z)|\bar{z}\rangle\langle\bar{z}| \quad \text { and } \quad H_{1}=\sum_{z \in\{0,1\}^{n}} E(z)|z\rangle\langle z|
$$

for eigenvalue functions $E(z)$ and $F(z)$ which are defined by the partitions $\mathcal{P}$ and $\mathcal{Q}$ and the sequences $\left(E_{j}\right)$ and $\left(F_{k}\right)$.

Let $s(t)$ be a smooth, nondecreasing, real function of time $t$ such that

$$
s:[0, T] \longrightarrow[0,1],
$$

with $s(0)=0$ and $s(T)=1$, for some final time $T$. It is clear that

$$
H_{\text {gen }}(t) \equiv(1-s(t)) H_{0}+s(t) H_{1}, \quad 0 \leq t \leq T,
$$

is a Hamiltonian having suitable form for adiabatic quantum algorithms solving problems for which $P_{0}$ contains the solutions. 
November $12, \quad 2018 \quad 5: 28 \quad$ WSPC/INSTRUCTION $\quad$ FILE

4 LAWRENCE M. IOANNOU and MICHELE MOSCA

\subsection{A general bound}

Let $\left|\psi_{\text {gen }}(t)\right\rangle$ be the state evolving under $H_{\text {gen }}(t)$, that is, we define the initial value problem

$$
\begin{aligned}
\frac{\partial}{\partial t}\left|\psi_{\text {gen }}(t)\right\rangle & =-1 H_{\text {gen }}(t)\left|\psi_{\text {gen }}(t)\right\rangle, \quad 0 \leq t \leq T \\
\left|\psi_{\text {gen }}(0)\right\rangle & =|u\rangle .
\end{aligned}
$$

Let $K$ be the index of the largest set $Q_{k}$ :

$$
\left|Q_{K}\right| \geq\left|Q_{k}\right| \quad \text { for all } k=0,1, \ldots, q(n) .
$$

Note

$$
H_{\text {gen }}(t)=(1-s(t))\left(F_{K} I-\sum_{k=0}^{q(n)}\left(F_{K}-F_{k}\right) \hat{Q}_{k}\right)+s(t) \sum_{z} E(z)|z\rangle\langle z| .
$$

Noting that

$$
|\bar{z}\rangle \equiv W|z\rangle=\frac{1}{\sqrt{N}} \sum_{x} \sum_{y}(-1)^{x \bullet y}|x\rangle\langle y|| z\rangle=\frac{1}{\sqrt{N}} \sum_{x}(-1)^{x \bullet z}|x\rangle,
$$

we have, dropping the time-dependence notation,

$$
\begin{aligned}
H_{\text {gen }}=(1-s) & \left(F_{K} I-\frac{1}{N} \sum_{x} \sum_{y}\left(\sum_{k=0}^{q(n)}\left(F_{K}-F_{k}\right) \sum_{z \in Q_{k}}(-1)^{z \bullet(x \oplus y)}\right)|x\rangle\langle y|\right) \\
& +s \sum_{z} E(z)|z\rangle\langle z| .
\end{aligned}
$$

Suppose $\left|\psi_{\text {gen }}(t)\right\rangle$ is expressed in the computational basis as $\left|\psi_{\text {gen }}(t)\right\rangle=$ $\sum_{z=0}^{N-1} \gamma_{z}(t)|z\rangle$. Clearly, the goal of the unitary evolution is to make $\sum_{w^{\prime} \in P_{0}}\left|\left\langle w^{\prime} \mid \psi_{\text {gen }}(T)\right\rangle\right|^{2}=\sum_{w^{\prime} \in P_{0}}\left|\gamma_{w^{\prime}}(T)\right|^{2}$ of constant (or just $1 / \operatorname{poly}(n)$ ) order so that we have a good probability of discovering one of the solutions $w^{\prime}$ by performing a measurement in the computational basis on the final state $\left|\psi_{\operatorname{gen}}(T)\right\rangle$. The Schrödinger equation implies

$$
\frac{\partial}{\partial t}\left|\psi_{\text {gen }}\right\rangle=-i \sum_{x}\left(\sum_{y}\left\langle x\left|H_{\text {gen }}\right| y\right\rangle \gamma_{y}\right)|x\rangle
$$


November $12, \quad 2018 \quad 5: 28 \quad$ WSPC/INSTRUCTION $\quad$ FILE

IoannouMosca:IJQI

For each solution $w^{\prime} \in P_{0}$,

$$
\begin{aligned}
& \frac{\partial}{\partial t}\left|\gamma_{w^{\prime}}\right|^{2} \\
= & 2 \operatorname{Re}\left(\left\langle w^{\prime}\left|\frac{\partial}{\partial t}\right| \psi_{\text {gen }}\right\rangle \cdot\left\langle w^{\prime} \mid \psi_{\text {gen }}\right\rangle^{*}\right) \\
= & 2 \sum_{y}\left\langle w^{\prime}\left|H_{\text {gen }}\right| y\right\rangle \operatorname{Im}\left(\gamma_{w^{\prime}} \gamma_{y}^{*}\right) \\
= & -2(1-s) \frac{1}{N} \sum_{y \neq w^{\prime}} \sum_{k=0}^{q(n)}\left(F_{K}-F_{k}\right)\left(\sum_{z \in Q_{k}}(-1)^{z \bullet\left(w^{\prime} \oplus y\right)}\right) \operatorname{Im}\left(\gamma_{w^{\prime}} \gamma_{y}^{*}\right) \\
= & -2(1-s) \frac{1}{N} \sum_{y} \sum_{k=0}^{q(n)}\left(F_{K}-F_{k}\right)\left(\sum_{z \in Q_{k}}(-1)^{z \bullet\left(w^{\prime} \oplus y\right)}\right) \operatorname{Im}\left(\gamma_{w^{\prime}} \gamma_{y}^{*}\right),
\end{aligned}
$$

where the last two lines hold because $\operatorname{Im}\left(\gamma_{w^{\prime}} \gamma_{w^{\prime}}^{*}\right)=0$. If the strings $z \in Q_{k}$ were somehow "random enough", we would expect the sums $\left(\sum_{z \in Q_{k}}(-1)^{z \bullet\left(w^{\prime} \oplus y\right)}\right)$ to be close to 0 . This would suggest that the derivative is small for an average problem instance. Without going into statistics about typical problem instances, we can only bound the size of these sums by $\left|Q_{k}\right|$. Using this bound for every $w^{\prime} \in P_{0}$ and using $\left|\sum_{y} \operatorname{Im}\left(\gamma_{w^{\prime}} \gamma_{y}^{*}\right)\right| \leq \sqrt{N}$ (which follows from applying the Cauchy-Schwarz inequality to the absolute value of the dot-product of the vector of all 1 's and the vector of $\operatorname{Im}\left(\gamma_{w^{\prime}} \gamma_{y}^{*}\right)$ values $)$ we get

$$
\left.\left|\sum_{w^{\prime} \in P_{0}} \frac{\partial}{\partial t}\right| \gamma_{w^{\prime}}\right|^{2} \mid \leq 2 \frac{\left|P_{0}\right|}{\sqrt{N}} \cdot \max _{k} F_{k} \cdot \operatorname{dim}\left(\operatorname{span}\left\{|\bar{z}\rangle: z \in Q_{K}\right\}^{\perp}\right) .
$$

Integrating from $t=0$ to $t=T$ as before, in order to lower-bound by a constant $c$ the total probability of finding a solution, we require

$$
T \geq \frac{c \sqrt{N}-\left|P_{0}\right| / \sqrt{N}}{2\left|P_{0}\right| \cdot \max _{k} F_{k} \cdot \operatorname{dim}\left(\operatorname{span}\left\{|\bar{z}\rangle: z \in Q_{K}\right\}^{\perp}\right)} .
$$

Assume that the number of solutions $\left|P_{0}\right|$ is small. Thus if the dimension of the subspace orthogonal to the largest eigenspace of $H_{0}$ is small, then the algorithm requires exponential time $T$.

\subsection{Searching with a more complex initial Hamiltonian}

We can use a similar argument to prove the analogous result for $H_{1}$. In this section, we illustrate the main ingredients of this similar argument by giving it in the simpler case where the final Hamiltonian is a one-dimensional projector. Thus, as explained below, we get an interesting variant of Farhi and Gutmann's optimality result in Ref. 14. We note that an argument similar to that in Ref. 9, based on the timereversibility of quantum mechanics, can be used to prove the following result; that is, the following result is implied by the "time-reversal" of the result in the previous 
section (or the results in Ref. 9 or Ref. 10, both of which consider one-dimensionalprojector initial Hamiltonians). Our short proof below need not appeal to timereversibility.

Consider the following problem: "What is the minimum time $T$ needed such that the Hamiltonian

$$
\begin{aligned}
H_{B}(t) & \left.\equiv H_{\text {gen }}(t)\right|_{p(n):=1,\left|P_{0}\right|:=1} \\
& =(1-s(t)) \sum_{z} F(z)|\bar{z}\rangle\langle\bar{z}|+s(t) E_{1}(I-|w\rangle\langle w|), \quad 0 \leq t \leq T
\end{aligned}
$$

(adiabatically) evolves the start state $|\psi(0)\rangle=|u\rangle$ to a final state $|\psi(T)\rangle$ that is close to $|w\rangle$ ?" Noting that

$$
|w\rangle\left\langle w\left|=\frac{1}{N} \sum_{x} \sum_{y}(-1)^{w \bullet(x \oplus y)}\right| \bar{x}\right\rangle\langle\bar{y}|,
$$

we have

$$
H_{B}=(1-s) \sum_{z} F(z)|\bar{z}\rangle\langle\bar{z}|+s E_{1}\left(I-\frac{1}{N} \sum_{x} \sum_{y}(-1)^{w \bullet(x \oplus y)}|\bar{x}\rangle\langle\bar{y}|\right) .
$$

Letting $|\psi(t)\rangle=\sum_{z} \beta_{z}(t)|\bar{z}\rangle$, the Schrödinger equation implies that

$$
\frac{\partial}{\partial t}|\psi\rangle=-i H_{B}|\psi\rangle=-1 \sum_{x} \sum_{y}\left\langle\bar{x}\left|H_{B}\right| \bar{y}\right\rangle \beta_{y}|\bar{x}\rangle
$$

Instead of bounding the rate at which probability amplitude flows into the solution state $|w\rangle$, we bound the rate at which it flows out of the start state $|u\rangle$. Noting that $\langle u \mid \psi(t)\rangle=\beta_{0}(t)$, we can derive that

$$
\frac{\partial}{\partial t}\left|\beta_{0}(t)\right|^{2}=-\frac{2 s E_{1}}{N} \sum_{y}(-1)^{w \bullet y} \operatorname{Im}\left(\beta_{y} \beta_{0}^{*}\right) .
$$

Using the Cauchy-Schwarz inequality we get the bound

$$
\left.\left|\frac{\partial}{\partial t}\right| \beta_{0}(t)\right|^{2} \mid \leq \frac{2 E_{1}}{\sqrt{N}}, \quad 0 \leq t \leq T .
$$

Noting that $\beta_{0}(0)=1$ and using

$$
\left|\beta_{0}(T)\right|^{2}-\left|\beta_{0}(0)\right|^{2}=\int_{0}^{T} \frac{\partial\left|\beta_{0}(t)\right|^{2}}{\partial t} d t \geq-\int_{0}^{T}\left|\frac{\partial\left|\beta_{0}(t)\right|^{2}}{\partial t}\right| d t
$$

gives

$$
\left|\beta_{0}(T)\right|^{2} \geq 1-\frac{2 E_{1} T}{\sqrt{N}}
$$

The following inequality holds: 16

$$
\left|\left\langle w \mid \psi_{B}(T)\right\rangle\right|^{2}+\left|\left\langle u \mid \psi_{B}(T)\right\rangle\right|^{2} \leq 1+|\langle u \mid w\rangle| .
$$


\begin{tabular}{|llll} 
November 12, & $2018 \quad 5: 28 \quad$ WSPC/INSTRUCTION & FILE
\end{tabular}

IoannouMosca:IJQI

LIMITATIONS OF SOME SIMPLE ADIABATIC QUANTUM ALGORITHMS

This implies

$$
\left|\left\langle w \mid \psi_{B}(T)\right\rangle\right|^{2} \leq \frac{2 E_{1} T}{\sqrt{N}}+\frac{1}{\sqrt{N}},
$$

which implies that the algorithm requires exponentially large time $T$ in order to compute $w$ with sufficiently high probability.

The result effectively bounds the power of any initial Hamiltonian that is diagonal in the Hadamard basis, in the presence of a final Hamiltonian that merely encodes the binary function $f(z)$ defining the generalized search problem (with unique solution $w$ ). We interpret this result in the context of Farhi and Gutmann's continuous-time search algorithm 14 Recall that their optimality result is that no Hamiltonian of the form $H_{D}(t)+H_{w}$ can solve the continuous-time search problem faster than $H_{u}+H_{w}$ if it must work faster on most problem instances $w \in\{0,1\}^{n}$. It follows from our result that no $H_{D}(t)$ that is restricted to being diagonal in the Hadamard basis can outperform $H_{u}$ on even one problem instance.

\section{Conclusion}

Combining the techniques of Sections 2.1 and 2.2, we can show that, in our generalized adiabatic scheme, both $H_{0}$ and $H_{1}$ must have at least two large mutuallyorthogonal eigenspaces if $T$ is to be subexponential in $n$.

\section{Acknowledgements}

LMI acknowledges the support of NSERC, the University of Waterloo, and EPSRC (UK). MM is supported by DTO-ARO, NSERC, CFI, ORDCF, CIAR, CRC, ORF, and Ontario-MTI.

\section{References}

1. E. Farhi, J. Goldstone, S. Gutmann, M. Sipser. "Quantum Computation by Adiabatic Evolution", http://subsubsection.lanl.gov/abs/quant-ph/0001106, 2000.

2. A. Messiah. Quantum Mechanics, Vol. II, Amsterdam: North Holland, New York: Wiley, 1976.

3. W. van Dam, U. Vazirani. Presented at QIP 2002, Yorktown Heights, NY, 2002.

4. E. Farhi, J. Goldstone, S. Gutmann. "A Numerical Study of the Performance of a Quantum Adiabatic Evolution Algorithm for Satisfiability", quant-ph/0007071, 2000.

5. E. Farhi, J. Goldstone, S. Gutmann, J. Lapan, A. Lundgren, D. Prada. "A Quantum Adiabatic Evolution Algorithm Applied to Random Instances of an NP-Complete Problem", Science, Vol. 292, 472, 2001.

6. E. Farhi, J. Goldstone, S. Gutmann. "Quantum Adiabatic Evolution Algorithms with Different Paths", MIT Report No. MIT-CTP-3297 (quant-ph/0208135), 2002.

7. T. Hogg. "Adiabatic quantum computing for random satisfiability problems", Physical Review A, Vol. 67, 022314, 2003.

8. Dorit Aharonov, Wim van Dam, Julia Kempe, Zeph Landau, Seth Lloyd, Oded Regev, in Proc. 45th Annual IEEE Symposium on Foundations of Computer Science (FOCS'04), San Diego, CA, 9-11 June (IEEE Computer Society, Washington D.C., 2004), pp. $42-51$. 
November $12, \quad 2018 \quad 5: 28 \quad$ WSPC/INSTRUCTION $\quad$ FILE

9. E. Farhi, J. Goldstone, S. Gutmann, D. Nagaj. "How to Make the Quantum Adiabatic Algorithm Fail", quant-ph/0512159.

10. Z. Wei and M. Ying. "Quantum adiabatic evolutions that can't be used to design efficient algorithms", quant-ph/0604077, 2006.

11. Marko Znidaric and Martin Horvat. "Exponential complexity of an adiabatic algorithm for an NP-complete problem", Phys. Rev. A. 73, 022329, 2006.

12. W. van Dam, M. Mosca and U. Vazirani, "How Powerful is Adiabatic Quantum Computation?", Proceedings of the 42nd Annual Symposium on Foundations of Computer Science (FOCS01), 279-287, Las Vegas, USA, 2001.

13. J. Roland and N. Cerf "Quantum Search by Local Adiabatic Evolution", Physical Review A, Vol. 65, 042308, 2002. Computers", Phys. Rev. A, 60(3):1956-1965, 1999.

14. E. Farhi, S. Gutmann. "An Analog Analogue of a Digital Quantum Computation", Physical Review A, 57, 2403, 1998.

15. M. R. Garey, D. S. Johnson. Computers and Intractability (A Guide to the Theory of NP-Completeness). W.H. Freeman and Company, New York, 1979.

16. L. M. Ioannou. "Continuous-time Quantum Algorithms: Searching and Adiabatic Computation", Masters Thesis, University of Waterloo, http://etheses.uwaterloo.ca, 2002. 